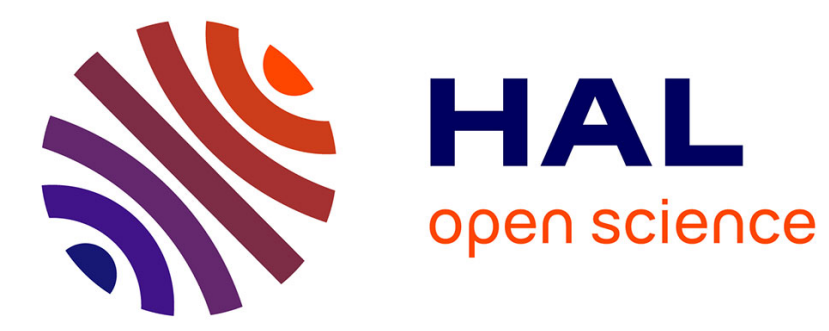

\title{
On isomorphic linear partitions in cubic graphs
}

Jean-Luc Fouquet, Henri Thuillier, Jean-Marie Vanherpe, Adam Pawel Wojda

\section{To cite this version:}

Jean-Luc Fouquet, Henri Thuillier, Jean-Marie Vanherpe, Adam Pawel Wojda. On isomorphic linear partitions in cubic graphs. Discrete Mathematics, 2009, 309 (22), pp.6425-6433. hal-00144338

\section{HAL Id: hal-00144338 \\ https://hal.science/hal-00144338}

Submitted on 2 May 2007

HAL is a multi-disciplinary open access archive for the deposit and dissemination of scientific research documents, whether they are published or not. The documents may come from teaching and research institutions in France or abroad, or from public or private research centers.
L'archive ouverte pluridisciplinaire HAL, est destinée au dépôt et à la diffusion de documents scientifiques de niveau recherche, publiés ou non, émanant des établissements d'enseignement et de recherche français ou étrangers, des laboratoires publics ou privés. 


\title{
On isomorphic linear partitions in cubic graphs
}

\author{
J-L. Fouquet, H. Thuillier, J-M. Vanherpe \\ L.I.F.O, University of Orléans, France \\ A.P. Wojda ${ }^{1}$ \\ Wydzial Matematyki Stosowanej Zaklad Matematyki Dyskretnej \\ A.G.H., Al. Mickiewicza 30, 30-059 Kraków, PL
}

\begin{abstract}
A linear forest is a graph that connected components are chordless paths. A linear partition of a graph $G$ is a partition of its edge set into linear forests and $l a(G)$ is the minimum number of linear forests in a linear partition. It is well known that $l a(G)=2$ when $G$ is a cubic graph and Wormald [17] conjectured that if $|V(G)| \equiv 0$ $(\bmod 4)$, then it is always possible to find a linear partition in two isomorphic linear forests. We give here some new results concerning this conjecture.
\end{abstract}

Key words: cubic graphs, linear-arboricity.

\section{Introduction.}

A linear-forest is a forest whose components are paths. The linear-arboricity of a graph $G$ introduced by Harary [12] is the minimum number of linear forests which partition its edge set (this number is denoted $l a(G)$ ). In this paper we consider cubic graphs, that is to say finite simple 3-regular graphs. It was shown by Akiyama, Exoo and Harary [1] that $l a(G)=2$ when $G$ is cubic. A partition of $E(G)$ into two linear forests $L_{B}$ and $L_{R}$ will be called a linear partition and we shall denote this linear partition by $L=\left(L_{B}, L_{R}\right)$.

For any cubic graph on $n \equiv 0(\bmod 4)$ vertices, it is an easy task to find a linear partition where we have the same number of paths in $L_{B}$ and in $L_{R}$ (see [2] for example).

1 The research of APW was partially supported by the AGH University of Science and Technology grant No 1142004 
Let $L=\left(L_{B}, L_{R}\right)$ be a linear partition of a cubic graph. For $j \in\{1, n-1\}$ let $n_{j}{ }^{B}$ be the number of paths of length $j$ in $L_{B}$ and define $n_{j}{ }^{R}$ in the same way for $L_{R}$ and let $\omega\left(L_{B}\right)\left(\omega\left(L_{R}\right)\right)$ be the number of components of $L_{B}$ ( $L_{R}$ respectively). In addition, we shall denote by $\mu\left(L_{B}\right)$ and $\mu\left(L_{R}\right)$ the mean lengths of paths in $L_{B}$ and $L_{R}$ while $l\left(L_{B}\right)$ and $l\left(L_{R}\right)$ will denote the maximum lengths of these paths.

Assume that $L=\left(L_{B}, L_{R}\right)$ is a linear partition such that $\omega\left(L_{B}\right)=\omega\left(L_{R}\right)$ (which implies that $n \equiv 0(\bmod 4))$ Theorem 1 below says, that, from statistical point of view, the two forests $L_{B}$ and $L_{R}$ are identical.

Theorem 1 [11] Let $L=\left(L_{B}, L_{R}\right)$ be a linear partition of a cubic graph $G$ such that $\omega\left(L_{B}\right)=\omega\left(L_{R}\right)$. Then

$$
\sum_{j=1}^{n-1}(j-3) n_{j}{ }^{B}=0 \quad \text { and } \quad \sum_{j=1}^{n-1}(j-3) n_{j}{ }^{R}=0 .
$$

Proof We know that $\omega\left(L_{B}\right)+\omega\left(L_{R}\right)=\frac{n}{2}$. Then,

$$
\mu\left(L_{B}\right)=\frac{\left|L_{B}\right|}{n-\left|L_{B}\right|}=\frac{n-\omega\left(L_{B}\right)}{\omega\left(L_{B}\right)}=\frac{2 \omega\left(L_{B}\right)+2 \omega\left(L_{R}\right)-\omega\left(L_{B}\right)}{\omega\left(L_{B}\right)}
$$

A similar equality holds for $L_{R}$ and we get,

$$
\mu\left(L_{B}\right)=3 \text { and } \mu\left(L_{R}\right)=3 .
$$

Considering $L_{B}$ we have,

$$
\sum_{j=1}^{n-1} j n_{j}{ }^{B}=\left|L_{B}\right|=\omega\left(L_{B}\right) \mu\left(L_{B}\right)
$$

Which leads to (the second equality being obtained when dealing with $L_{R}$ )

$$
\sum_{j=1}^{n-1} j n_{j}{ }^{B}=3 \omega\left(L_{B}\right) \text { and } \sum_{j=1}^{n-1} j n_{j}{ }^{R}=3 \omega\left(L_{R}\right)
$$

We obviously have $\sum_{j=1}^{n-1} n_{j}{ }^{B}=\omega\left(L_{B}\right)$ and $\sum_{j=1}^{n-1} n_{j}{ }^{R}=\omega\left(L_{R}\right)$. Hence,

$$
\sum_{j=1}^{n-1}(j-3) n_{j}{ }^{B}=0 \quad \text { and } \quad \sum_{j=1}^{n-1}(j-3) n_{j}{ }^{R}=0 .
$$

In fact, a conjecture of Wormald [17] goes further in that direction. 
Conjecture 2 [17] Let $G$ be a cubic graph with $|E(G)| \equiv 0(\bmod 2)$ (or equivalently $|V(G)| \equiv 0(\bmod 4))$. Then there exists a linear partition $L=$ $\left(L_{B}, L_{R}\right)$ of $E(G)$ such that $L_{B}$ and $L_{R}$ are isomorphic linear forests.

Theorem 10 below (see [5] and independently [17]) implies that Conjecture 2 is true for Jaeger's graphs (see definition 6). Up to our knowledge, it is even the only known class for which the Wormald conjecture is proved.

Our purpose, in that paper, is to give some new results concerning this conjecture.

\section{Preliminaries}

Assume that $G$ is a cubic graph and let $M$ be a matching transversal of the odd cycles, that is a matching which intersects the edge-set of every odd cycle. Since $G \backslash M$ is bipartite, we can colour $V(G)$ in two colours blue and red accordingly to the bipartition of $G \backslash M$ (let $B$ and $R$ these two sets of vertices). An edge of $E(G)$ is said to be mixed when one end is blue while the other is red. Hence, the edges of $G \backslash M$ are mixed while $M$ is partitioned into three sets (some of them, possibly empty)

$$
M=M_{B}+M_{R}+M^{\prime}
$$

$M_{B}$ is the set of edges with two ends in $B, M_{R}$ the set of edges its two ends in $R$ and $M^{\prime}$ is a set of mixed edges.

A strong matching $C$ in a graph $G$ is a matching $C$ such that there is no edge of $E(G)$ connecting any two edges of $C$, or, equivalently, such that $C$ is the edge-set of the subgraph of $G$ induced on the vertex-set $V(C)$. Note that $M_{B}$ and $M_{R}$ induce strong matchings in $G$ since the neighbours of an edge in $M_{B}$ (respectively $M_{R}$ ) are joined to this edge by a mixed edge.

Theorem 3 A cubic graph is 3-edge colourable if and only if there is a partition of its vertex set into two sets, $B$ and $R$ and a perfect matching $M$ such that every edge in $G-M$ is mixed.

Proof Let $G$ be a cubic 3-edge colourable graph. Any colour of a 3-edge colouring of $G$ induces a perfect matching $M$, and the two others colours induce a graph whose components are even cycles. Let us colour these cycles in $B$ and $R$ alternately. Hence every edge lying on these cycles is mixed.

Conversely, assume that $G$ has a perfect matching $M$ and a partition of its vertex set into $B$ and $R$ such that every edge in $G-M$ is mixed. Let us 
consider the 2-factor of $G$ obtained in deleting $M$. Since every edge outside $M$ is mixed, this 2-factor is even, which means that $G$ is 3-edge colourable.

Remark 4 Under conditions of Theorem 3 we certainly have the same number of vertices in $B$ and in $R$, since every edge of the 2-factor $G-M$ is mixed. When considering $M=M_{B}+M_{R}+M^{\prime}$ we have $\left|M_{B}\right|=\left|M_{R}\right|$ since every mixed edge of $M$ uses a vertex in each colour.

\subsection{Definitions}

As usually, for any undirected graph $G$, we denote by $V(G)$ the set of its vertices and by $E(G)$ the set of its edges and we consider usually that $|V(G)|=$ $n$ and $|E(G)|=m$. If $F \subseteq E(G), V(F)$ is the set of vertices which are incident with some edges of $F$. For any path $P$ we shall denote by $l(P)$ the length of $P$, that is to say the number of its edges. A vertex of a path $P$ distinct from an end-vertex is said to be an internal vertex.

Let $L=\left(L_{B}, L_{R}\right)$ be a linear partition of a cubic graph, since every vertex of $G$ is either end-vertex of a maximal path of $L_{B}$ or end-vertex of a maximal path of $L_{R}$, we have

$$
\omega\left(L_{B}\right)+\omega\left(L_{R}\right)=\frac{|V(G)|}{2} .
$$

Let $M$ be a perfect matching of a cubic graph $G$. An $M$-alternating path (or alternating path when no confusion is possible) is a path $v=v_{0} v_{1} v_{2} \ldots v_{2 k+1}=$ $w$ such that any edge $v_{i} v_{i+1}$ (where $i$ is odd) is an edge of $M$. We shall say that two distinct vertices $v$ and $w$ are at alternating distance $2 k+1(k \geq 0)$ whenever a shortest alternating path joining these two vertices has length $2 k+1$. An alternating cycle is an alternating path where the two extremities are joined by an edge of $M$.

Definition 5 Let $G$ be a cubic 3-edge colourable graph with a perfect matching $M$ given in Theorem 3 by a 3 -edge colouring. We shall say that a partition of $M$ in $M_{B}, M_{R}$ and $M^{\prime}$ is an $M$-associated partition (or associated partition for short).

Definition 6 We shall say that a cubic graph $G$ is a Jaeger's graph whenever $G$ contains a perfect matching which is a union of two disjoint strong matchings. A Jaeger's matching is a perfect matching which is the union of two strong matchings. 
Assume that $G$ is a Jaeger's graph and let $M_{B}$ and $M_{R}$ be the two strong matchings which partition a Jaeger's matching $M$ of $G$. Let us colour with blue the vertices which are ends of edges in $M_{B}$ and red those which are ends of edges in $M_{R}$. It is an easy task to see that the remaining edges are mixed. Hence $G$ is 3-edge colourable and, as pointed out in Remark 4 we have $\left|M_{B}\right|=\left|M_{R}\right|=|M| / 2$. The associated partition $M=M_{B}+M_{R}+M^{\prime}$ is such that $M^{\prime}$ is empty.

In his thesis [14] Jaeger called these cubic graphs equitable and pointed out that the above two colouring of their vertices leads to a balanced colouring defined by Bondy [6].

Definition 7 An odd linear forest is a linear forest in which each path has odd length.

Aldred and Wormald [3] proved that a cubic graph $G$ can be factored into two odd linear forests if and only if $G$ is 3-edge coloured (i.e. $\chi^{\prime}(G)=3$ ).

\subsection{Associated linear construction}

Assume that we are given a cubic 3-edge colourable graph together with an associated partition $M=M_{B}+M_{R}+M^{\prime}$. Let us fix an arbitrary orientation to the cycles of $G \backslash M$. To each vertex $v$ of $V(G)$ we can associate an edge $o(v)$ of $E(G) \backslash M$ such that $v$ is the origin of $o(v)$ with respect to the chosen orientation of the cycle through $v$. It will be convenient to denote by $s(v)$ ( successor of $v$ ) the end of $o(v)$ in that orientation and by $p(v)$ its predecessor. We can colour $o(v)$ in blue or red accordingly to the colour of $v . M_{B}$ being coloured with blue and $M_{R}$ with red, we get hence a larger set $C L_{B}$ of edges coloured with blue (and $C L_{R}$ of edges coloured with red). It is easily seen that the $C L_{B}$ and $C L_{R}$ are linear-forests where each maximal unicoloured path has length 1 or 3. Moreover each edge of $M_{B} \cup M_{R}$ is the central edge of a path of length 3 . At this point, the only edges which are not coloured are the edges of $M^{\prime}$ and we do not know how we can affect a colour to these edges in order to get a linear partition of $E(G)$.

Definition 8 We shall refer to the above construction of $C L_{B}$ and $C L_{R}$ when an associated partition is given as the associated linear construction and we denote this construction $C L=\left(C L_{B}, C L_{R}\right)$.

Proposition 9 Let us colour at random any edge of $M^{\prime}$ with blue or red and let $M_{R}^{\prime}$ and $M_{B}^{\prime}$ the two subsets of $M^{\prime}$ so obtained. Then the connected components of the subgraphs induced by $V\left(C L_{R} \cup M_{R}^{\prime}\right)$ (the red components) and $V\left(C L_{B} \cup M_{B}^{\prime}\right)$ (the blue components) are alternating cycles or alternating paths. 
Proof Since the construction of $C L_{B}$ and $C L_{R}$ is obtained in colouring alternatively red and blue the edges of an even 2 -factor each vertex is incident to at least one edge in each colour. Hence the maximum degree in each colour is 2. A vertex of degree 2 in a red component (blue component) is incident to a mixed edge of the 2 -factor and an edge of $M_{R} \cup M_{R}^{\prime}\left(M_{B} \cup M_{B}^{\prime}\right.$ respectively). Hence the connected components in each colour are alternating path and alternating cycles as claimed.

Theorem 10 [5], [17] A cubic graph $G$ has a linear partition $L=\left(L_{B}, L_{R}\right)$ such that each path has length 3 if and only if $G$ is a Jaeger's graph.

Proof : Suppose that $G$ has a linear partition $L=\left(L_{B}, L_{R}\right)$ with maximum lengths $l\left(L_{B}\right) \leq 3$ and $l\left(L_{R}\right) \leq 3$. Since $\omega\left(L_{B}\right)+\omega\left(L_{R}\right)=\frac{|V(G)|}{2}$, and $|E(G)|=3 \frac{|V(G)|}{2}$ each path in $L_{B}$ and $L_{R}$ have length exactly 3. Let $M_{B}$ (resp. $M_{R}$ ) be the set of the middle edges of the paths of $L_{B}$ (resp. $L_{R}$ ). It is an easy task to check that $M_{B}$ and $M_{R}$ are strong matchings and $\left|M_{B}\right|=\left|M_{R}\right|$. Moreover $M=M_{B} \cup M_{R}$ is a perfect matching and $G$ is a Jaeger's graph .

Conversely, let us suppose that $G$ is a Jaeger's graph and let $M=M_{B}+M_{R}$ be an associated partition. Since $M^{\prime}$ is empty, in using the associated linear construction above, we have coloured every edge of $G$ and each unicoloured path has length 3.

\section{Associated partition with extra conditions}

When a cubic 3-edge colourable graph and an associated partition $M=$ $M_{B}+M_{R}+M^{\prime}$ are given, it is rather natural to impose some extra condition on $M^{\prime}$ in order to extend Theorem 10 and obtain a larger class of graphs for which Conjecture 2 holds. In that section we consider the case where the connected components of $V\left(M^{\prime}\right)$ are paths of length 1 or 3. Starting from the associated linear construction $C L=\left(C L_{B}, C L_{R}\right)$ we try to colour $M^{\prime}$ (ie partition $M^{\prime}$ in $M_{B}^{\prime}$ and $\left.M_{R}^{\prime}\right)$ expecting that the partition of $E(G)\left(C L_{B} \cup M^{\prime} B, C L_{R} \cup M_{R}^{\prime}\right)$ so obtained is a linear partition with nice properties.

Recall that, as pointed out before (see Proposition 9), the connected components of $C L_{B}$ and $C L_{R}$ are paths of length 1 or 3. Moreover, the number of paths of length 3 is the same in $C L_{B}$ and $C L_{R}$ as well as the number of paths of length 1.

In this section, we shall assume that $G$ is a cubic 3-edge colourable graph and $M=M_{B}+M_{R}+M^{\prime}$ an associated partition that the components of $V\left(M^{\prime}\right)$ 
are paths of lengths 1 or 3 . In this conditions, it is always possible to partition $M^{\prime}$ into 2 strong matchings, namely $M_{B}^{\prime}$ and $M_{R}^{\prime}$, the edges of $M_{B}^{\prime}$ will be said blue while the edges of $M_{R}^{\prime}$ are red.

Lemma 11 For any partition of $M^{\prime}$ into two strong matchings $M_{B}^{\prime}$ and $M_{R}^{\prime}$, the partition of $E(G)\left(C L_{B} \cup M_{B}^{\prime}, C L_{R} \cup M_{R}^{\prime}\right)$ is a linear partition.

Proof By Proposition 9, the connected components of $C L_{B} \cup M_{B}^{\prime}$ are alternating paths and cycles. Let $b r$ be an edge of $M_{B}^{\prime}$ such that $b \in B$ and $r \in R$ and let $r^{\prime}=s(b)$. Since $G \backslash M$ contains only mixed edges $r^{\prime}$ is a red vertex. Observe that $o\left(r^{\prime}\right)$ is a red edge while the edge of $M$ incident to $r^{\prime}$ in $G$, say $e$, cannot belong to $M_{B}^{\prime}$ since $M^{\prime}$ is a strong matching. Moreover, $e$ having a red end cannot belong to $C L_{B}$, consequently $e$ belongs to $C L_{R} \cup M_{R}^{\prime}$. Thus, among the three edges incident to $r^{\prime}$, only $o(b)$ is in $C L_{B} \cup M_{B}^{\prime}$ which means that the connected component of $C L_{B} \cup M_{B}^{\prime}$ containing $b r$ is an alternating path. Since we can use the same argument for any edge in $M^{\prime},\left(C L_{B} \cup M_{B}^{\prime}, C L_{R} \cup M_{R}^{\prime}\right)$ is a linear partition.

Lemma 12 There is a partition of $M^{\prime}$ into two strong matchings $M_{B}^{\prime}$ and $M_{R}^{\prime}$ such that there is no new path of length 3 in $C L_{B} \cup M_{B}^{\prime}$ and $C L_{R} \cup M_{R}^{\prime}$.

Proof Let $b r \in M_{B}^{\prime}$ (with $b \in B$ and $r \in R$ ). Assume that $b r$ is contained in a new path of length 3 of $C L_{B} \cup M_{B}^{\prime}$. This path is certainly $s(b) \operatorname{brp}(r)$. The edge of $M$ incident to $p(r)$ say $e$ is in $C L_{R} \cup M_{R}^{\prime}$ since $p(r)$ is an endpoint of a blue path of length 3 , more precisely $e \in M_{R}^{\prime}$ for otherwise the edge $p(r) r$ would be in $C L_{R}$. Consequently the edge of $M$ incident to $s(b)$ say $e^{\prime}$ belongs to $M_{R}$, as a matter of fact if on the contrary we have $e \in M_{R}^{\prime}$ we would have in the subgraph induced with $V\left(M^{\prime}\right)$ a path of length greater than 3 containing the edges $e, p(r) r, r b, b s(b), e^{\prime}$ a contradiction. From now on $e$ is denoted $p(r) r^{\prime}$ $\left(r^{\prime} \in R\right)$.

The path $r^{\prime} p(r) r b s(b)$ being a component of the subgraph induced with $V\left(M^{\prime}\right)$ we have that the edge of $M$ which is incident to $p(b)$ is in $M_{R}$, similarly $s(r)$ is incident to an edge of $M_{B}, p\left(b^{\prime}\right)$ to an edge of $M_{B}$ and $s\left(b^{\prime}\right)$ to an edge of $M_{B}$. Hence, $b$ is an endpoint of a path of length 3 of $C L_{R}$ say $P_{1}, p(r)$ is an endpoint of a path of length 3 in $C L_{R}$ and $r^{\prime}$ is an endpoint of a path of length 3 in $C L_{B}$, say $P_{2}$. We set :

$$
M_{B}^{\prime}=M_{B}^{\prime}-b r+p(r) r^{\prime}, M_{R}^{\prime}=M_{R}^{\prime}-p(r) r^{\prime}+b r
$$

Observe that $M_{B}^{\prime}$ and $M_{R}^{\prime}$ remain to be strong matchings. In addition $s(r)$ becomes an endpoint of a path in $C L_{B} \cup M_{R}^{\prime}$ of length at least 5 which contains $P_{1}, b s(b)$ is a path of length 1 in $C L_{B} \cup M_{B}^{\prime}$ while $r$ becomes an endpoint of a 
path of length at least 5 in $C L_{B} \cup M_{B}^{\prime}$ which contains $P_{2}$ and $r^{\prime} s\left(b^{\prime}\right)$ is a path of length 1 in $C L_{R} \cup M_{R}^{\prime}$.

This operation can be iterated for each new path of length 3 in $C L_{B} \cup M_{B}^{\prime}$ or in $C L_{R} \cup M_{R}^{\prime}$ and the result follows.

Remark 13 Observe that when $M^{\prime}$ itself is a strong matching, then Lemma 12 holds for all partitions of $M^{\prime}$ into $M_{B}^{\prime}$ and $M_{R}^{\prime}$.

Lemma $14 A$ path of length 5 in $C L_{B} \cup M_{B}^{\prime}\left(C L_{R} \cup M_{R}^{\prime}\right)$ is obtained by connecting a path of length 1 and a path of length 3 of $C L_{B}\left(C L_{R}\right.$ respectively).

Proof A path in $C L_{B} \cup M_{B}^{\prime}$ is an alternating path. Since $M_{B}^{\prime}$ is a strong matching, such a path of length 5 contains at most one edge of $M_{B}^{\prime}$. In the same way this path contains at most one edge of $M_{B}$ ( $M_{B}$ being a strong matching). Hence a path of length 5 must contains one edge of $M_{B}^{\prime}$ and one edge of $M_{B}$. These edge of $M_{B}$ leads to a path of length 3 in $C L_{B}$ and the edge of $M_{B}$ connects this path of length 3 to a path of length 1 of $C L_{B}$ as claimed.

Lemma $15 A$ path of length 7 in $C L_{B} \cup M_{B}^{\prime}$ (resp. $C L_{R} \cup M_{R}^{\prime}$ ) is obtained by connecting two paths of length 1 of $C L_{B}$ (resp. $C L_{R}$ ) to one path of length 3 of $C L_{B}$ (resp. $\left.C L_{R}\right)$, moreover each path of the linear partition $L=\left(C L_{B} \cup\right.$ $\left.M_{B}^{\prime}, C L_{R} \cup M_{R}^{\prime}\right)$ has odd length at most 7 .

Proof Let $b_{1} r_{1}$ be an edge of $M_{B}^{\prime}\left(b_{1} \in B, r_{1} \in R\right)$. Let us set $r_{2}=s\left(b_{1}\right)$. The edge of $M$ incident to $r_{2}$ cannot be in $M_{B}^{\prime}$ since $M_{B}^{\prime}$ is a strong matching nor in $M_{B}$ since $r_{2}$ is a red vertex, thus $r_{2}$ is one end of the path of $C L_{B} \cup M_{B}^{\prime}$ containing $b_{1} r_{1}$.

Let $b_{2}=p\left(r_{1}\right)$ and $r_{3}=p\left(b_{2}\right)$, obviously $b_{2} \in B, r_{3} \in R, b_{2} r_{1}$ is a blue edge and $r_{3} b_{2}$ is a red one. Consider in $G$ the edge of $M$ incident to $b_{2}$, say e. $M_{B}^{\prime}$ being a strong matching, the edge $e$ cannot belong to $M_{B}^{\prime}$. Moreover, the edge $e$ has a blue end, namely $b_{2}$, and thus cannot belong to $M_{R}$. Hence $e$ is in $M_{B} \cup M_{R}^{\prime}$.

If $e \in M_{R}^{\prime}$ the path $b_{2} r_{1} b_{1} r_{2}$ is a path of length 3 in $C L_{B} \cup M_{B}^{\prime}$ and we are done.

From now on $e$ is in $M_{B}$ and will be denoted $b_{2} b_{3}\left(b_{3} \in B\right)$ and $s\left(b_{3}\right)$ will be denoted $r_{4}$, we have $r_{4} \in R$. Let $e^{\prime}$ be the edge of $M$ incident to $r_{4}$. Since $r_{4}$ is a red end of $e^{\prime}, e^{\prime}$ cannot be in $M_{B}$. If $e^{\prime}$ is a member of $M_{R} \cup M_{R}^{\prime}$ we are done since $e^{\prime}$ and $o\left(r_{4}\right)$ both are in $C L_{R} \cup M_{R}^{\prime}$ and the path of $C L_{B} \cup M_{B}^{\prime}$ containing $b_{1} r_{1}$ is reduced to a path of length 5 , namely $r_{2} b_{1} r_{1} b_{2} b_{3} r_{4}$. 
Suppose now that $e^{\prime} \in M_{B}^{\prime}$, Let us denote $e^{\prime}$ as $r_{4} b_{4}\left(b_{4} \in B\right)$ and $s\left(b_{4}\right)$ as $r_{5}$. But now, the edge of $M$ which contains $r_{5}$ cannot be in $M_{B}$ since $r_{5}$ is a red vertex nor in $M_{B}^{\prime}$ since $M_{B}^{\prime}$ is a strong matching. Hence $P=$ $\left\{r_{2}, b_{1}, r_{1}, b_{2}, b_{3}, r_{4}, b_{4}, r_{5}\right\}$ induces a path of length 7 and this path is obtained in connecting two paths of length 1 to a path of length 3 of $C L_{B}$.

When we decide to put an edge of $M^{\prime}$ in $M_{B}^{\prime}$ or $M_{R}^{\prime}$ we can create only a path of length 3 or a path of length 5 or a path of length 7 . Hence we have obtained an odd linear partition where each path has length at most 7 .

In [16] Thomassen, answering positively a conjecture in [5], showed that any cubic graph can be provided with a linear partition where each path has length at most 5. Aldred and Wormald [3], obtained before 9 instead of of 5 , but using their method we can prove that a cubic 3-edge colourable graph has an odd linear partition with every path of odd length at most 7 . In our case we get more precise information about the distribution of paths of length 7 when we have a stronger condition on the matching $M^{\prime}$, namely when $M^{\prime}$ itself is a strong matching.

Theorem 16 Let $G$ be cubic 3-edge colourable graph and an associated partition $M=M_{B}+M_{R}+M^{\prime}$. Assume that $M^{\prime}$ is a strong matching. Then there is an odd linear partition $L=\left(L_{B}, L_{R}\right)$ of $E(G)$ where each path has length $1,3,5$ or 7 such that $\left|n_{7}^{B}-n_{7}^{R}\right| \leq 1$.

Proof From Lemmas 11 and 15, we know that in partitioning $M^{\prime}$ in $M_{B}^{\prime}$ and $M_{R}^{\prime}$ we get a linear partition $\left(L_{B}, L_{R}\right)$ where each path has odd length at most 7 .

If for that linear partition $\left|n_{7}^{B}-n_{7}^{R}\right| \leq 1$ we are done, w.l.o.g. let us suppose that $n_{7}^{B}>n_{7}^{R}+1$. Then pick an edge $b r \in M_{B}^{\prime}$ which is on a path of length 7 in $C L_{B} \cup M_{B}^{\prime}$ and put this edge in $M_{R}^{\prime}$. Hence $M_{B}^{\prime}$ becomes $M_{B}^{\prime} \backslash b r$ while $M_{R}^{\prime}$ becomes $M_{R}^{\prime}+b r$. Applying the preceding reasoning in Lemma 15 we get an odd path of length at most 7 entirely contained in $C L_{R} \cup M_{R}^{\prime}$. Since we have lost a path of length 7 in $C L_{B} \cup M_{B}^{\prime}$, in the new linear partition so obtained $\left|n_{7}^{B}-n_{7}^{R}\right|$ is strictly smaller than before. We can thus perform our exchange as long as $\left|n_{7}^{B}-n_{7}^{R}\right| \leq 1$ and we get our result.

Independently of Theorem 16, if we choose the strong matchings $M_{B}^{\prime}$ and $M_{R}^{\prime}$ in such a way that Lemma 12 holds and if we suppose that we have the same number of paths of length 7 (we do not claim that it is always possible) it is reasonable to think that we have an isomorphic linear partition.

Theorem 17 Let $G$ be a cubic 3-edge colourable graph, $M=M_{B}+M_{R}+$ $M^{\prime}$ an associated partition where $V\left(M^{\prime}\right)$ induces a subgraph whose connected components are paths of length 1 or 3 . Let $L=\left(L_{B}, L_{R}\right)$, where $L_{B}=C L_{B} \cup$ $M_{B}^{\prime}$ and $L_{R}=C L_{R} \cup M_{R}^{\prime}$, be an odd linear partition obtained in partitioning $M^{\prime}$ in $M_{B}^{\prime}$ and $M_{R}^{\prime}$ into two strong matchings such that 
- there is no new path of length 3 in $L=\left(L_{B}, L_{R}\right)$

- $n_{7}^{B}=n_{7}^{R}$

- $\omega\left(L_{B}\right)=\omega\left(L_{R}\right)$

Then $L=\left(L_{B}, L_{R}\right)$ is an isomorphic odd linear partition.

Proof Let $p_{1}^{B}=p_{1}^{R}$ be the number of paths of length 1 and let be $p_{3}^{B}=$ $p_{3}^{R}$ the number of paths of length 3 in $C L_{B}$ and $C L_{R}$ (the two sets of an associated linear construction leading to our linear partition $\left.L=\left(L_{B}, L_{R}\right)\right)$. Since $\omega\left(L_{B}\right)=\omega\left(L_{R}\right)$, by Theorem 1 we have:

$$
-2 n_{1}^{B}+0 n_{3}^{B}+2 n_{5}^{B}+4 n_{7}^{B}=-2 n_{1}^{R}+0 n_{3}^{R}+2 n_{5}^{R}+4 n_{7}^{R}=0
$$

since we have the same number of paths of length 7 , we get

$$
-n_{1}^{B}+n_{5}^{B}=-n_{1}^{R}+n_{5}^{R}
$$

Since there is no path of length 3 in $L=\left(L_{B}, L_{R}\right)$, we have from Lemmas 13 and 14

$$
\begin{gathered}
p_{1}^{B}=n_{1}^{B}+n_{5}^{B}+2 n_{7}^{B} \\
p_{1}^{R}=n_{1}^{R}+n_{5}^{R}+2 n_{7}^{R}
\end{gathered}
$$

and hence, taking into account that $n_{7}^{B}=n_{7}^{R}$

$$
n_{1}^{B}+n_{5}^{B}=n_{1}^{R}+n_{5}^{R}
$$

Indeed, each path of length 5 uses one path of length 1 of $C L_{B}$ while, as pointed out in Lemma 15, a path of length 7 needs to use two such paths.

From Equations (2) and (3) we get $n_{1}^{B}=n_{1}^{R}$ and $n_{5}^{B}=n_{5}^{R}$.

Since we have $p_{3}^{B}=p_{3}^{R}$, there is no new path of length 3 in $L$ and each path of length 5 or 7 uses exactly one path of length 3 in $C L$ (see Lemmas 14 and $15)$, we necessarily have $n_{3}^{B}=n_{3}^{R}$. Hence the linear partition $L=\left(L_{B}, L_{R}\right)$ is isomorphic as claimed.

Theorem 18 Let $G$ be a cubic 3-edge colourable graph on $n \equiv 0$ (4) vertices and let $M=M_{B}+M_{R}+M^{\prime}$ be an associated partition. Assume that for any two edges e and $e^{\prime}$ in $M^{\prime}$ the shortest alternating path joining these two edges has length at least 5. Then $G$ has an odd isomorphic linear partition.

Proof Since $M^{\prime}$ is even $(n \equiv 0(4))$ we can choose to partition $M^{\prime}$ in $M_{B}^{\prime}$ and $M_{R}^{\prime}$ in such a way that $\left|M_{B}^{\prime}\right|=\left|M_{R}^{\prime}\right|$. Let $L=\left(L_{B}=C L_{B}+M_{B}^{\prime}, L_{R}=\right.$ $\left.C L_{R}+M_{R}^{\prime}\right)$ be the odd linear partition so obtained from Theorem 16. Let $b_{1} r_{1}$ be an edge of $M_{B}^{\prime}$, we have seen in Lemma 15 that when we create a path of length 7 in $C L_{B} \cup M_{B}^{\prime}$ containing $b_{1} r_{1}$ (let $P=\left\{r_{2}, b_{1}, r_{1}, b_{2}, b_{3}, r_{4}, b_{4}, r_{5}\right\}$ 
this path), the edge $r_{4} b_{4}$ is itself in $M_{B}^{\prime}$. In that case the alternating distance between $b_{1} r_{1}$ and $r_{4} b_{4}$ is thus 3 . Since we have supposed that no two edges in $M^{\prime}$ are joined by an alternating path of length less than 5 , that means that this case does not occur and no path of length 7 can be created. Hence each edge of $M_{B}^{\prime}$ leads to a path of length 5 (recall that no path of length 3 can be created by Lemma 12) in $C L_{B} \cup M_{B}^{\prime}$ as well as each edge of $M_{R}^{\prime}$ in $C L_{R} \cup M_{R}^{\prime}$. Since $\left|M_{B}^{\prime}\right|=\left|M_{R}^{\prime}\right|$ we have hence $\omega\left(L_{B}\right)=\omega\left(L_{R}\right)$ and the result follows from Theorem 17

Corollary 19 Let $G$ be a cubic 3-edge colourable graph on $n \equiv 0$ (4) having a 2 -factor of triangles. Then $G$ has an odd isomorphic linear partition.

Proof Assume that $G$ is three edge coloured and let $M=M_{B}+M_{R}+M^{\prime}$ be an associated partition. It is an easy matter to see that each triangle contains an edge of $M_{B}$ or $M_{R}$ while exactly one edge connecting this triangle to another one is also in $M$. Hence the three edges of each triangle are affected in the associated linear construction either to $C L_{B}$ or to $C L_{R}$. The edges of $M^{\prime}$ are edges connecting some triangles of our 2-factor (each triangle being incident to at most one edge of $M^{\prime}$ ). If $M^{\prime}$ is empty, $G$ is a Jaeger's graph and we are done. $\left|M^{\prime}\right|$ being even, let $x y$ and $x^{\prime} y^{\prime}$ be two distinct edges of $M^{\prime}$, we want to show that their alternating distance is at least 5 .

Assume that $x$ is contained in the triangle $x u v$ and $y$ in $y w t$ while $x^{\prime}$ is contained in $x^{\prime} u^{\prime} v^{\prime}$ and $y^{\prime}$ in $y^{\prime} w^{\prime} t^{\prime}$. A shortest alternating path joining $x y$ to $x^{\prime} y^{\prime}$ begins with $w u v$ or $x v u$ or ywt or $y t w$. In the same way, it must ends with $v^{\prime} u^{\prime} x^{\prime}$ or $u^{\prime} v^{\prime} x^{\prime}$ or $t^{\prime} w^{\prime} t^{\prime}$ or $w^{\prime} t^{\prime} y^{\prime}$. Since each triangle is incident to at most one edge of $M^{\prime}$, such a shortest alternating path has length at least 5 . The conclusion follows from theorem 18 .

In [11] it is shown that a cubic graph having a 2-factor of squares is a Jaeger's graph and, hence, can be provided with an isomorphic linear partition. As a step towards Conjecture 2, it could be interesting to generalize these results by considering $k$-uniform 2 -factors (each cycle has length $k$ for a fixed $k \geq 5$ ).

\section{Graphs with strong chromatic index 5}

A strong edge colouring of a graph $G$ is a partition of its edge set into strong matchings. Let $\chi_{S}(G)$ (strong chromatic index) denote the minimum integer $k$ for which $E(G)$ can be partitioned into $k$ strong matchings of $G$. This notion was introduced in [10] and [9] while [7] is the usual reference for the origin of this problem. When dealing with cubic graphs, we have immediately that $\chi_{S}(G) \geq 5$. We know that $\chi_{S}(G) \leq 10$ (see [4] and [13]) for cubic graphs 
in general and $\chi_{S}(G) \leq 9$ (see [15]) when considering cubic bipartite graphs (answering thus positively to conjectures appearing in [9] and [8]).

The class of cubic graphs satisfying $\chi_{S}(G)=5$ (as Petersen's graph, Dodecahedron and the graphs associated to $C_{60}$ the molecule of the well known fulleren, a polyhedra on 60 vertices whose faces are 12 cycles on 5 vertices and 20 cycles on 6 vertices) is of particular interest. A simple counting argument leads to $|V(G)| \equiv 0$ (10). By the way this implies that $\chi_{S}(G) \geq 6$ when $|V(G)| \not \equiv 0$ (10) which gives us easy counterexamples to a conjecture in [8] asserting that $\chi_{S}(G)=5$ when $G$ is a cubic bipartite graph with girth sufficiently large.

Proposition 20 [9] Let $G$ be a cubic graph with $\chi_{S}(G)=5$. Then the spanning subgraph of $G$ obtained by considering 3 colours is an induced subgraph of $k K_{1,3}$ and an induced subgraph of cycles without chord of length $\equiv 0$ (6). The sum of the lengths of these cycles being $6 k$.

Proof Assume that we have coloured $E(G)$ with the five (strong) colours $\{1,2,3,4,5\}$. Let us remark that each edge of $G$ is incident to each colour. W.l.o.g. we consider the 3 colours $\{1,2,3\}$. Since we use 5 strong matchings to colour the edges of $G$, each vertex is certainly incident to at least one colour in $\{1,2,3\}$. Hence these 3 colours leads to a spanning subgraph of $G$. Assume that $v$ is a vertex incident to the 3 colours. $v$ is the center of a $K_{1,3}$ coloured with $\{1,2,3\}$. This subgraph is induced in $G$ otherwise we have two neighbors of $v$ joined by an edge. In that case $G$ contains a triangle and it is easy to see that we need at least 6 strong matchings to colour the edges incident to the 3 vertices of a triangle, a contradiction. Let us remark that each neighbor of $v$ is incident to 2 edges coloured with 4 and 5 . If we consider two distinct $K_{1,3}$ 's centered in $v$ and $w$ then an edge joining a neighbor $v^{\prime}$ of $v$ to a neighbor $w^{\prime}$ of $w$ would be incident to two edges of colour 4 or 5 , contradiction. Hence, the set of $K_{1,3}$ coloured with $\{1,2,3\}$ is an induced subgraph of $G$.

Assume now that $v_{1} v_{2}$ is coloured $1, v_{2} v_{3}$ is coloured 2 while $v_{1}, v_{2}, v_{3}$ are not the center of one of the $K_{1,3}$ 's coloured with $\{1,2,3\} . v_{2}$ is not incident with 3 and $v_{3}$ is not incident with 1 . Hence $v_{3}$ is certainly incident with 3 . Let $v_{4}$ be the end of the edges of colour 3 incident with $v_{3} . v_{4}$ is not the center of a $K_{1,3}$ coloured with $\{1,2,3\} \cdot v_{4}$ must be incident with 1 leading to a new vertex $v_{5}$ etc... We construct in that way a cycle whose edges are alternatively coloured 1,2 and 3. We can check that this cycle has no chord (otherwise a chord would be incident to two edges with the same colour). Moreover this cycle is even, since each edge is incident to an edge coloured with 4 and the other with 5 . These two colours are alternated along the cycle. Each such cycle has length multiple of 6 as claimed. The edges of colours 4 and 5 have one end on a $K_{1,3}$ and the other on one of the cycles. Hence the sum of the lengths of our cycles must be exactly $6 k$, the number of edges of colour 4 or 5 incident 
to the pendent vertices of the set of $K_{1,3}$ 's.

Theorem 21 Let $G$ be a cubic graph with $\chi_{S}(G)=5$. Let $\mathcal{K}$ be the set of $K_{1,3}$ induced by 3 colours in a 5 -strong edge colouring of $G$ and let $\mathcal{C}$ be the set of corresponding cycles. Assume that $|\mathcal{K}|=k$. Then $G$ has a linear partition $L=\left(L_{B}, L_{R}\right)$ such that

- $L_{B}$ is a set of $p$ paths of length $6, p+2 q$ paths of length 2 and $q$ paths of length 3

- $L_{R}$ is a set of $q$ paths of length $6, q+2 p$ paths of length 2 and $p$ paths of length 3

for any two integers $p$ and $q$ such that $p+q=k$

Proof Assume that we have partition $E(G)$ with the five (strong) colours $\{1,2,3,4,5\}$. W.l.o.g. we consider that $\mathcal{K}$ and $\mathcal{C}$ is obtained in using the 3 strong matchings $\{1,2,3\}$. Let us colour alternatively the edges of each cycle of $\mathcal{C}$ in red and blue and let us colour the edges of 4 with red and those of 5 with blue. At this point, we remark that each blue connected component is a path of length 2 as well as each red connected component. Moreover each vertex of $\mathcal{C}$ is the end vertex of a red path (or a blue) path and the interior vertex of a blue path (or a red path respectively). Let us remark that the only edges which are not coloured in red or blue are the edges in $\mathcal{K}$. For each $K_{1,3} \in \mathcal{K}$, the three pendent vertices are the end of a red path of length 2 (and a blue path of length 2), the other end being on $\mathcal{C}$. Assume that $v$ is the center of a $K_{1,3} \in \mathcal{K}$ and let $v_{1}, v_{2}$ and $v_{3}$ be its 3 (distinct) neighbors. Let us put $v_{1} v$ and $v v_{2}$ in blue while $v v_{3}$ is set in red. We get hence a blue path of length 6 and a red path of length 3. In addition, we have 2 red paths of length 2 ending in $v_{1}$ and $v_{2}$ and a blue path of length 2 ending in $v_{3}$. We could had put $v_{1} v$ and $v v_{2}$ in red and $v v_{3}$ in blue, obtaining thus a red path of length 6 and a blue path of length 3, 2 blue paths of length 2 ending in $v_{1}$ and $v_{2}$ with a red path of length 2 ending in $v_{3}$. We operate in the same way on each $K_{1,3} \in \mathcal{K}$.

Since, in that process, we are connecting two distinct paths of length two, with the same colour, each having exactly one end on $\mathcal{C}$, while a path of length 2 is extended to a path of length 3 , without changing any previous coloured edge (in $\mathcal{C}$ as well as edges in 4 and 5 ), we are sure that any path involved in that operation cannot be extended from one $K_{1,3} \in \mathcal{K}$ to another one. Moreover, for each $K_{1,3}$, we are free to choose the colour leading to a path of length 6 . Hence we can decide to create $p$ blue paths of length 6 and $p$ red paths of length 3 when operating on $p K_{1,3} \in \mathcal{K}$ and to create $q$ red paths of length 6 and $q$ blue paths of length 3 with the $q$ remaining $K_{1,3}$ 's. The result follows. 
Observe that the isomorphic linear forests considered in Theorem 21 are not necessarily odd.

Corollary 22 Let $G$ be a cubic graph with $\chi_{S}(G)=5$ and having a number of vertices multiple of 20 . Then $G$ can be partitioned into two isomorphic linear forests.

Proof In that case $|\mathcal{K}|=k$ is even. From theorem 21 we consider $p=q=\frac{k}{2}$ and we get the result.

Recall that a cubic planar graph is a multi-k-gon [9] (with $3 \leq k \leq 5$ ) if all its faces have length multiple of $k$. We know that multi-3-gons and multi-4-gons satisfy Conjecture 2 since they are Jaeger's graphs (see [5]). Multi-5-gons are not Jaeger's graphs in general, however we can show that they do have an isomorphic linear partition.

Corollary 23 Let $G$ be a multi-5-gon. Then $G$ can be partitioned into two isomorphic linear forests.

Proof In [10], it is proved that the strong chromatic index of a multi-5-gon is 5 and its number of vertices is multiple of 20 . The result follows from corollary 22

\section{$5 \quad$ Near Jaeger's graphs}

We have seen that whenever an associated partition $M=M_{B}+M_{R}+M^{\prime}$ is such that $V\left(M^{\prime}\right)$ induces components of length 1 or 3 , we can find, in some cases, an isomorphic linear partition, extending thus the previous known result on Jaeger's graphs. An other way to explore is to assume an upper bound of the number of edges in $M^{\prime}$. In Theorem 24 below we consider the simple case where $M^{\prime}$ has only two edges.

Theorem 24 Let $G$ be a cubic 3-edge colourable graph on $n \equiv 0$ (4) vertices. Assume that we can find a 3-edge colouring with an associated partition $M=$ $M_{B}+M_{R}+M^{\prime}$ where $M^{\prime}$ has exactly two edges. Then there exists a linear partition $L=\left(L_{B}, L_{R}\right)$ of $E(G)$ such that $L_{B}$ and $L_{R}$ are isomorphic odd linear forests.

Proof Let $C L=\left(C L_{B}, C L_{R}\right)$ be the linear construction associated to $M$ (see Definition 8). In deleting $M$ we are left with an even 2-factor $\mathcal{C}$ whose vertices are alternatively coloured blue and red, let $B$ be the set of blue vertices and 
$R$ be the set of red ones. Let us denote the two mixed edges of $M^{\prime}$ as $b_{1} r_{1}$ and $b_{2} r_{2}\left(b_{1}, b_{2} \in B\right.$ and $\left.r_{1}, r_{2} \in R\right)$.

We may assume that $b_{1}$ and $r_{2}$ are adjacent as well as $b_{2}$ and $r_{1}$; for otherwise the subgraph induced by $\left\{b_{1}, r_{1}, b_{2}, r_{2}\right\}$ is either a $P_{4}$ or a $2 K_{2}$, we set $M_{B}^{\prime}$ and $M_{R}^{\prime}$ in such a way that there is no new path of length 3 in $L_{B}=C L_{B} \cup M_{B}^{\prime}$ nor in $L_{R}=C L_{R} \cup M_{R}^{\prime}$ (see Lemma 12), by Lemma 15 we know that $n_{7}^{B}=n_{7}^{R}=0$ and thus Theorem 17 applies.

Hence $b_{1} r_{2}, b_{2} r_{1} \in E(G)$. In addition, we can suppose that in following the orientation given to each cycle of $\mathcal{C}$ we have $r_{2}=s\left(b_{1}\right)$ and $b_{2}=s\left(r_{1}\right)$ : the contrary implies that the edges $b_{1} r_{2}$ and $b_{2} r_{1}$ are on the same cycle of $\mathcal{C}$, namely $b_{1} r_{2} \ldots b_{2} r_{1} \ldots b_{1}$, in this case we replace the mixed edges $b_{1} r_{1}$ and $b_{2} r_{2}$ with $b_{1} r_{2}$ and $r_{1} b_{2}$ we consider the linear construction associated to the perfect matching $M_{B}+M_{R}+b_{1} r_{2}+r_{1} b_{2}$, we get another 2-factor $\mathcal{C}^{\prime}$ such that $b_{1} r_{2}$ and $r_{1} b_{2}$ are not on the same cycle of $\mathcal{C}^{\prime}$.

We denote $p\left(b_{1}\right)$ as $r_{3}, s\left(r_{2}\right)$ as $b_{3}, p\left(r_{1}\right)$ as $b_{4}$ and $s\left(b_{2}\right)$ as $r_{4}\left(b_{3}, b_{4} \in\right.$ $\left.B, r_{3}, r_{4} \in R\right)$. Hence we have the oriented paths $r_{3} b_{1} r_{2} b_{3}$ and $b_{4} r_{1} b_{2} r_{4}$ while $b_{1} r_{2}, b_{4} r_{1}, b_{2} r_{4} \in M_{B}$ and $r_{3} b_{1}, r_{2} b_{3}, r_{1} b_{2} \in M_{R}$.

We know that $r_{3}$ is the end of an edge of $M_{R}$, namely $r_{3} r_{3}^{\prime}$, moreover $b_{4}$ is the end of an edge $b_{4} b^{\prime} 4$ of $M_{B}$. The linear construction leads to paths of length 3 ending in this vertices. More precisely, $r_{3}$ is the end of a path $P_{1}$ of $C L_{B}$ while $r_{3}^{\prime}$ is the end of a path $P_{2}$ of $C L_{B}$. In the same way we have paths $P_{3}$ and $P_{4}$ of $C L_{R}$ ending in $b_{4}$ and $b_{4}^{\prime}$.

Case 1: Assume that $P_{1} \neq P_{2}$ and $P_{3} \neq P_{4}$.

In this case (see figure 1 ) we set $L_{B}=C L_{B}-b_{4} b_{4}^{\prime}+r_{3} r_{3}^{\prime}+r_{2} b_{2}$ and $L_{R}=$ $C L_{R}-r_{3} r_{3}^{\prime}+b_{4} b_{4}^{\prime}+b_{1} r_{1}$. It follows that $L_{B}$ and $L_{R}$ have both the same number of paths of length 3 , one path of length 7 and two paths of length 1 .

Case 2: $P_{1}=P_{2}$ or $P_{3}=P_{4}$.

W.l.o.g we assume that $P_{1}=P_{2}=r_{3} b_{5} b_{5}^{\prime} r_{3}^{\prime}\left(b_{5}, b_{5}^{\prime} \in B\right)$. The edge $r_{3} r_{3}^{\prime}$ is the central edge of a path $Q$ of length 3 in $C L_{R}$, the vertex $b_{5}$ is an endpoint of a path $R$ in $C L_{R}$ while the edge $b_{4} b_{4}^{\prime}$ is the central edge of a path $S$ of length 3 in $C L_{B}$.

In this case ( see Figure 2) we set $L_{B}=C L_{B}-b_{5} r_{3}+r_{3} r_{3}^{\prime}+b_{1} r_{1}$ and $L_{R}=$ $C L_{R}-r_{3} r_{3}^{\prime}+b_{5} r_{3}+r_{2} b_{2}$.

We have transformed $Q$ into two paths of length 1, $R$ into a path of length 5 ending with $b_{5} r_{3} b_{1}$ and these two paths are in $L_{R}$ while $P_{1}$ is replaced by $b_{5} b_{5}^{\prime} r_{3}^{\prime} r_{3}$. The path $S$ is transformed into a path of length ending with $r_{1} b_{1} r_{2}$. 

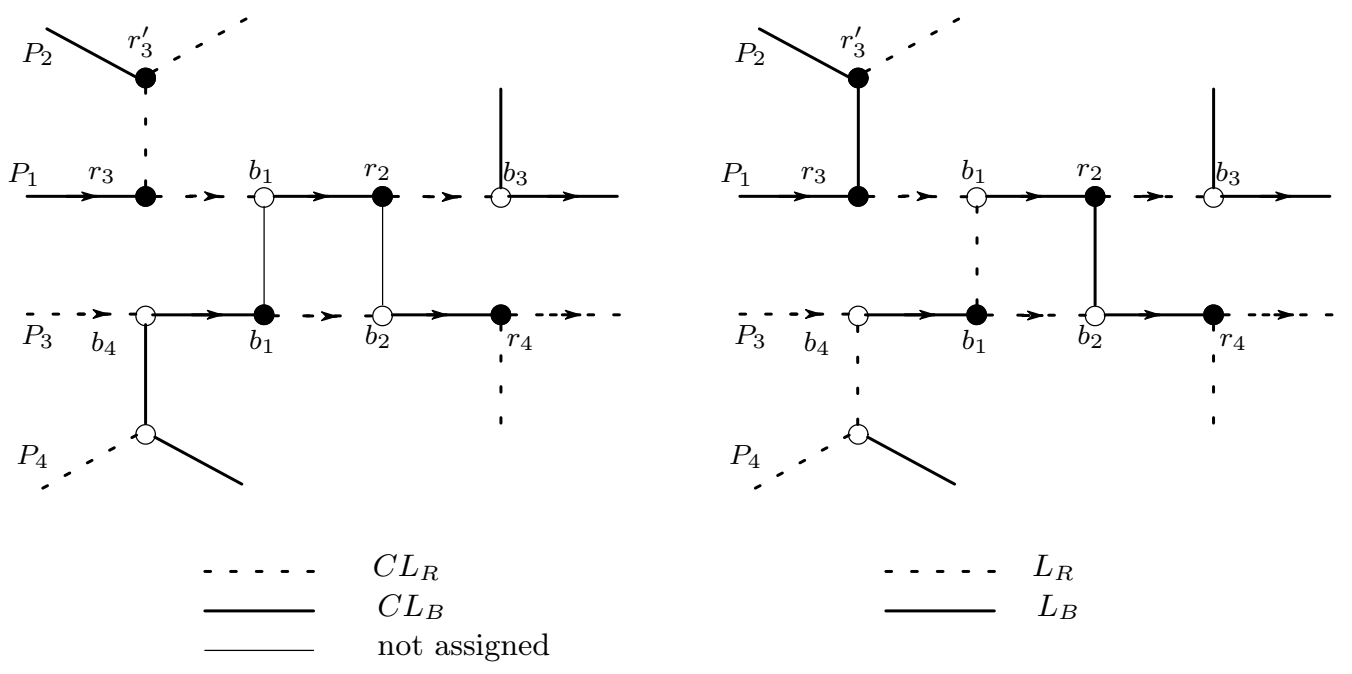

Fig. 1. Isomorphic linear partitions case 1

Since $r_{2} b_{2} \in L_{R}$ we have created a path of length 3 , namely $r_{1} b_{2} r_{2} b_{3}$ in $L_{R}$ which takes the place of $Q$. Consequently, $L_{B}$ and $L_{R}$ have both the same number of paths of length 3 , one path of length 5 and one path of length 1.

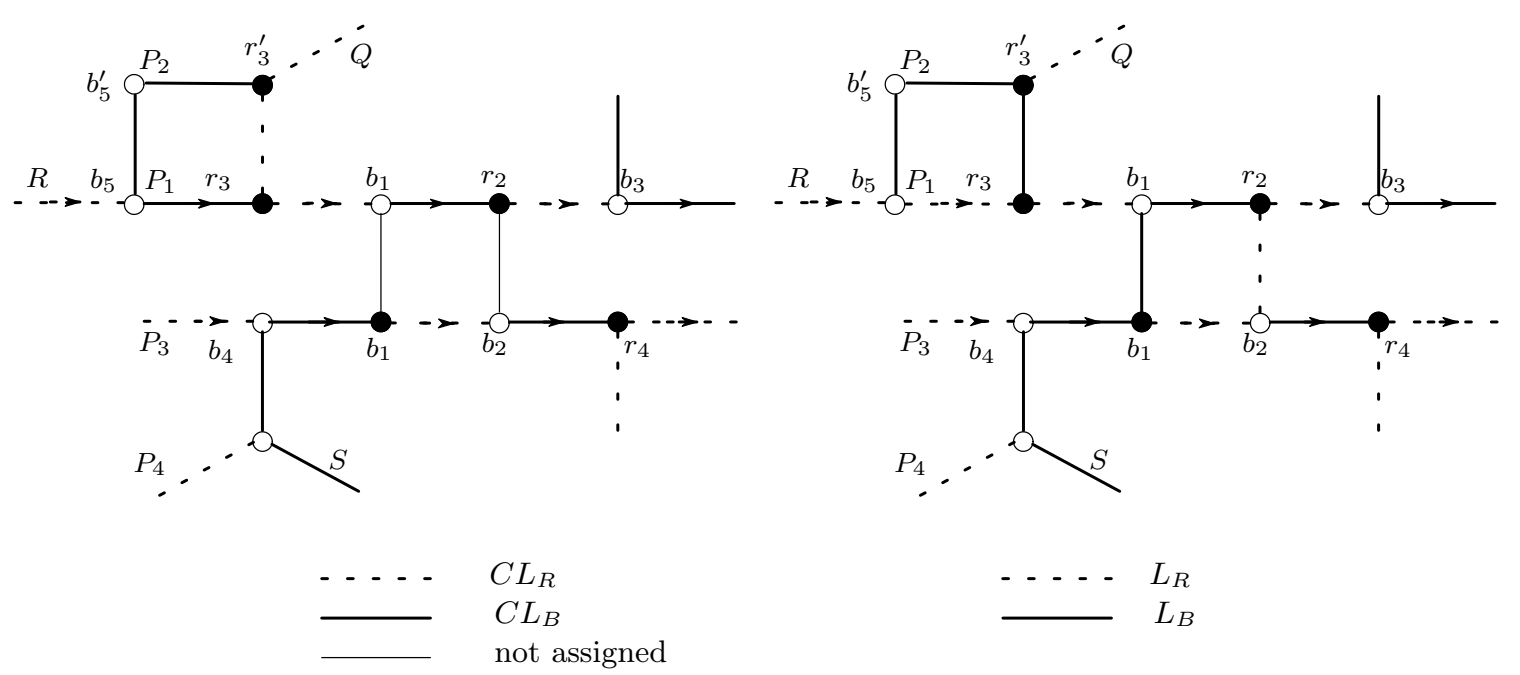

Fig. 2. Isomorphic linear partitions case 2

Either in case 1 and case 2 both forests $L_{B}$ and $L_{R}$ have the same number of paths of length 1 , of paths of length 3 , of paths of length 5 and of paths of length 7 thus $L_{B}^{\prime}$ and $L_{R}^{\prime}$ are isomorphic odd linear forests. 


\section{References}

[1] J. Akiyama, G. Exoo, and F. Harary. Covering and packing in graphs iii. Cyclic and Acyclic Invariant, Math. Slovaca, 30:405-417, 1980.

[2] R.E.L. Aldred, Bill Jackson, D. Lou, and A. Saito. Partitioning regular graphs into equicardinal linear forests. Discrete Mathematics, 88:1-9, 1991.

[3] R.E.L. Aldred and N.C. Wormald. More on the linear k-arboricity of regular graphs. Australas. J. Combin., 18:97-104, 1998.

[4] L.D. Andersen. The strong chromatic index of a cubic graph is at most 10 . Discrete Math, 1992.

[5] J-C. Bermond, J-L. Fouquetand M. Habib, and B. Peroche. On linear karboricity. Discrete Mathematics, 52:123-132, 1984.

[6] J. A. Bondy. Balanced colourings and the four colour conjecture. In Proc. of the Am. math. Soc, volume 33 of 2, pages 231-244, 1972.

[7] P. Erdôs and J. Nešetr̃il. Irregularities of partitions. Ed. G. Halász and V.T.Sós, pages 162-163, 1989.

[8] R.J. Faudree, A. Gyárfás, and Z. Tuza. The strong chromatic index of graphs. Ars Combinatoria, 29:205-211, 1990.

[9] J-L. Fouquet and J-L. Jolivet. Strong edge-colourings of graphs and applications to multi-k-gons. Ars Combinatoria, 16(A):141-150, 1983.

[10] J-L. Fouquet and J-L. Jolivet. Progress in Graph Theory, chapter Strong edgecolouring of cubic planar graphs, pages 247-264. Academic Press, j.a. bondy and u.s.r. murty edition, 1984.

[11] J-L. Fouquet, H. Thuillier, J-M. Vanherpe, and A.P. Wojda. On linear arboricity of cubic graphs. preprint, 2006.

[12] F. Harary. Covering and packing in graphs i. Ann. New York Acad. Sci., 175:198-205, 1970.

[13] P. Horák, H. Qing, and W.T. Trotter. Induced matchings in cubic graphs. J. Graph Theory, 17:151-160, 1993.

[14] F. Jaeger. étude de quelques invariants et problèmes d'existence en théorie de graphes. Thèse d'état, IMAG, Grenoble, 1976.

[15] A. Steger and Min li Yu. On induced matchings. Discrete Mathematics, 120:291295, 1993.

[16] C. Thomassen. Two-coloring the edges of a cubic graph such that each monochromatic component is a path of length at most 5. J. Comb. Theory B, 75:100-109, 1999.

[17] N. Wormald. Problem 13. Ars Combinatoria, 23(A):332-334, 1987. 